\title{
Structural and functional motor cortex asymmetry in unilateral lower limb amputation with phantom limb pain
}

\author{
K. Pacheco-Barrios ${ }^{a, b}$, CB. Pinto ${ }^{a}$, FG. Saleh Velez ${ }^{a, c}$, D. Duarte ${ }^{a, g}$, ME. Gunduz $^{a}$, M. Simis ${ }^{\text {d }}$, \\ AC. Lepesteur Gianlorenco ${ }^{\mathrm{a}}$, JL. Barouh ${ }^{\mathrm{a}}$, D. Crandell ${ }^{\mathrm{e}}$, M. Guidetti ${ }^{\mathrm{f}}$, L. Battistella ${ }^{\mathrm{d}}$, F. Fregni $^{\mathrm{a}, *}$

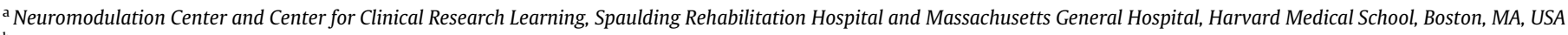 \\ ${ }^{\mathrm{b}}$ Universidad San Ignacio de Loyola, Vicerrectorado de Investigación, Unidad de Investigación para la Generación y Síntesis de Evidencias en Salud, Lima, Peru \\ ' University of Chicago Medical Center, Department of Neurology, University of Chicago, Chicago, IL, USA \\ ${ }^{\mathrm{d}}$ Hospital das Clinicas HCFMUSP, Faculdade de Medicina, Universidade de Sao Paulo, Sao Paulo, Brazil \\ e Spaulding Rehabilitation Hospital, Harvard Medical School, Boston, MA, USA \\ ${ }_{\mathrm{f}}^{\mathrm{f}}$ Università degli Studi di Milano, Dipartimento di scienze della Salute, "Aldo Ravelli" Center for Neurotechnolgy and Experimental Brain Therapeutics, Milano, Italy \\ ${ }^{\mathrm{g}}$ Department of Psychiatry and Behavioural Neurosciences, McMaster University, Canada.
}

\section{A R T I C L E I N F O}

\section{Article history:}

Accepted 1 June 2020

Available online 15 July 2020

\section{Keywords:}

Phantom limb pain

Cortical mapping

Magnetic resonance imaging

\section{H I G H L I G H T S}

- There is an asymmetrical reorganization of the motor cortex in patients with phantom limb pain

- We found disorganized and shifted hand cortical representation in the affected hemisphere.

- This reorganization is not associated with pain intensity.

\begin{abstract}
A B S T R A C T
Objective: The role of motor cortex reorganization in the development and maintenance of phantom limb pain (PLP) is still unclear. This study aims to evaluate neurophysiological and structural motor cortex asymmetry in patients with PLP and its relationship with pain intensity.

Methods: Cross-sectional analysis of an ongoing randomized-controlled trial. We evaluated the motor cortex asymmetry through two techniques: i) changes in cortical excitability indexed by transcranial magnetic stimulation (motor evoked potential, paired-pulse paradigms and cortical mapping), and ii) voxel-wise grey matter asymmetry analysis by brain magnetic resonance imaging.

Results: We included 62 unilateral traumatic lower limb amputees with a mean PLP of 5.9 (SD =1.79). We found, in the affected hemisphere, an anterior shift of the hand area center of gravity $(23 \mathrm{~mm}, 95 \% \mathrm{CI} 6$ to $38, \mathrm{p}=0.005$ ) and a disorganized and widespread representation. Regarding voxel-wise grey matter asymmetry analysis, data from 21 participants show a loss of grey matter volume in the motor area of the affected hemisphere. This asymmetry seems negatively associated with time since amputation. For TMS data, only the ICF ratio is negatively correlated with PLP intensity $(r=-0.25, p=0.04)$.

Conclusion: There is an asymmetrical reorganization of the motor cortex in patients with PLP, characterized by a disorganized, widespread, and shifted hand cortical representation and a loss in grey matter volume in the affected hemisphere. This reorganization seems to reduce across time since amputation. However, it is not associated with pain intensity.

Significance: These findings are significant to understand the role of the motor cortex reorganization in patients with PLP, showing that the pain intensity may be related with other neurophysiological factors, not just cortical reorganization.
\end{abstract}

(c) 2020 International Federation of Clinical Neurophysiology. Published by Elsevier B.V. All rights reserved.

* Corresponding author at: Neuromodulation Center and Center for Clinical Research Learning, Spaulding Rehabilitation Hospital and Massachusetts General Hospital, Harvard Medical School, 96 13th Street, Charlestown, Boston, MA, United States.

E-mail address: Fregni.Felipe@mgh.harvard.edu (F. Fregni).

\section{Introduction}

Phantom limb pain (PLP) is a neuropathic pain syndrome defined as the feeling of painful sensations perceived along the area of the absent limb (Flor, 2002). Its prevalence can be as high 
as $80 \%$ in amputees (Richardson, 2009), leading to severe deterioration of the independence level and quality of life in this population (Sinha et al., 2011). In addition, PLP can be frequently associated with phantom limb sensation (PLS) - any non-painful sensation within the area of the absent limb - and as well with residual limb pain - persistent pain localized within the residual limb (RLP) or stump area that does not resolve despite complete stump healing (Nikolajsen and Jensen, 2001).

Despite the high prevalence and impact in the quality of life (van der Schans et al., 2002), the underlying mechanism of PLP remains not fully known (Richardson, 2009).

There are several theories trying to delineate factors associated with its development and differences in pain intensity, among them, increasing evidence suggests a major role of the cortical reorganization of sensory and motor cortex in the genesis and maintenance on phantom pain (Birbaumer et al., 1997, Flor et al., 1995, Flor et al., 2006, Preißler et al., 2017). Two non-invasive techniques, transcranial magnetic stimulation (TMS) and brain magnetic resonance imaging (MRI), have been used to elucidate the neural alterations caused by amputation - especially related to structural and neurophysiological asymmetries - and their correlation with pain intensity (MacIver et al., 2008, Nardone et al., 2019, Preißler et al., 2017).

Cortical representation changes are frequently observed in PLP patient studies; however, mixed results are observed. The cortical remapping theory states that after limb amputation, the central control area of the amputated limb becomes deafferented, and the potential unmasking of previously silent connections leads to functional and cortical reorganization (Flor et al., 1995, Grüsser et al., 2001, Montoya et al., 1998, Ramachandran et al., 1992).

In this regard, Flor et al. (1995) and Raffin et al. (2016) showed that cortical changes (maladaptive reorganization) were closely associated with PLP: they reported a shift of nearby lip representation area into the deafferented hand region in upper-limb amputees. These results concluded that the larger the shift of the mouth representation into the deafferented zone, the greater the level of PLP. On the contrary, Makin et al. (2013) and Kikkert et al. (2018) demonstrated opposite findings, stating that a reduction in interregional functional connectivity is the more likely underlying mechanism of pain presence and this is, on the other hand, associated with a preservation of brain structure organization (Kikkert et al., 2018, Makin et al., 2013). Moreover, studies report loss of gray matter in the motor cortex area, primary somatosensory cortex, and the anterior cingulate cortex (ACC) (Draganski et al., 2006, Jiang et al., 2015, Preißler et al., 2012) after amputation. This evidence together pointed to the conclusion that the relationship of this cortical reorganization and PLP is unclear.

The same mixed results are observed in transcranial magnetic stimulation (TMS) studies, even though most of the studies showed alterations in motor cortex excitability, including the first study published by Cohen showing increased motor evoked potentials (MEP) and higher number of excitable cortical sites for the muscles immediately proximal of the stump (Cohen et al., 1991); others challenged these findings and showed no significant difference in the map areas or the shift of their locations (Gagné et al., 2011).

In view of these controversial findings, larger studies evaluating neurophysiological and structural changes in patients with PLP are needed. So far, there is no understanding as to why and how cortical reorganization following amputation relates to pain presence or intensity, as well as with pain-related outcomes.

In this study, we aimed to evaluate neurophysiological and structural motor cortex asymmetry in traumatic lower-limb amputees suffering from PLP to (i) describe motor cortex changes resulting from the amputation indexed by TMS (MEP, paired-pulse, and cortical mapping) and brain MRI (grey matter differences); (ii) identify neurophysiological and structural factors related to PLP intensity.

\section{Methods}

\subsection{Study design}

This is a cross-sectional analysis of an ongoing two-center randomized, double-blinded, placebo-controlled clinical trial (NCT02487966) (Pinto et al., 2016). The trial was reviewed and approved by Partners Institutional Review Board - Spaulding Rehabilitation Hospital and the University of Sao Paulo Institutional Review Board. Written informed consent was obtained from all enrolled participants before all trial procedures. For detailed information on the study design and full methodology, see Pinto et al. (2016).

\subsection{Participants}

This study includes all participants that underwent a baseline visit from the beginning of the trial until August of 2019. Sixtytwo participants were included in the neurophysiological data (TMS) and 21 in the MRI-VBM analysis (not all the subjects underwent MRI procedure).

Participants were considered eligible to participate in the study if they fulfilled the following inclusion criteria: (1) amputees ( $>18$ years old) with a unilateral traumatic lower limb amputation were eligible only if they were experiencing PLP for more than 3 months prior to the beginning of the study, (2) with an average above 4 on a Visual analog scale (VAS) for pain from 0 to 10 , and they were (3) able to provide informed consent. Subjects with any contraindication to tDCS and TMS were not enrolled, as well as subjects with a history of neurosurgery, chronic pain before the amputation, or unexplained fainting or loss of consciousness. Other exclusion criteria included subjects that underwent mirror therapy (MT) within 3 months prior to enrolment, severe depression, uncontrolled epilepsy, and unstable medical conditions (Pinto et al., 2016).

Sixty-two subjects were included: 37 from the Sao Paulo site and 25 from the Boston site. The sample was $32.26 \%$ male, and the mean age of the participants was $43.05 \pm 15.18$ years - the median time since amputation was 18 months (IQR: 7-56) at study enrollment. The mean BDI and BAI were $9.08 \pm 8.65$ and $9.66 \pm 8.28$, respectively. Further clinical data related to the amputation are provided in Table 1 .

\subsection{Clinical and demographic variables}

\subsubsection{Demographic questionnaire}

We used a standardized form to collect demographic variables, including age, gender, ethnicity, and participation site

\subsubsection{Amputation-related variables}

We collected this information by the Groningen Questionnaire after Arm Amputation, adapted for lower limb amputation (Kooijman et al., 2002). This instrument included questions on PLP, RLP, and Phantom limb sensation (PLS). Similar to our previous study (Münger et al., 2020), we obtained data on the amputation side, level of amputation, time since amputation, pain prior to the amputation, opioid use, previous treatment, and whether it has been effective, PLP/RLP/PLS frequency, PLS quality and types (Münger et al., 2020).

\subsubsection{Beck depression index (BDI) and beck anxiety index (BAI)}

"Assesses the psychological well-being encompassing diverse symptoms of anxiety and depression as well as their severity. Further demographics and medical history were assessed during the 
Table 1

Clinical and demographic characteristics $(n=62)$.

\begin{tabular}{|c|c|}
\hline \multicolumn{2}{|l|}{ Side of amputation } \\
\hline Left & 35 (56.45\%) \\
\hline Right & $27(43.55 \%)$ \\
\hline \multicolumn{2}{|l|}{ Level of amputation } \\
\hline Above the knee & $32(51.61 \%)$ \\
\hline Below the knee & $30(48.39 \%)$ \\
\hline \multicolumn{2}{|l|}{ Pain prior to the amputation } \\
\hline Yes & $38(61.29 \%)$ \\
\hline No & $24(38.71 \%)$ \\
\hline \multicolumn{2}{|l|}{ Opioid intake } \\
\hline Yes & $12(19.35 \%)$ \\
\hline No & $50(80.65 \%)$ \\
\hline \multicolumn{2}{|l|}{ Gabapentin intake } \\
\hline Yes & $12(20.00 \%)$ \\
\hline No & $50(80.00 \%)$ \\
\hline \multicolumn{2}{|l|}{ Pregabalin intake } \\
\hline Yes & $4(7.00 \%)$ \\
\hline No & $58(93.00 \%)$ \\
\hline \multicolumn{2}{|l|}{ Non-opioid analgesics intake } \\
\hline Yes & $15(25.00 \%)$ \\
\hline No & 47 (75.00\%) \\
\hline \multicolumn{2}{|l|}{ Protheses use ( $n=43$ ) } \\
\hline Yes & $25(58.10 \%)$ \\
\hline No & $18(41.90 \%)$ \\
\hline \multicolumn{2}{|l|}{ Phantom limb pain frequency } \\
\hline At least one time per day & $33(56.00 \%)$ \\
\hline Less than one time per day & $29(44.00 \%)$ \\
\hline
\end{tabular}

consent visit and included in the present study" (Münger et al., 2020).

\subsubsection{Visual analogue scale}

As in our previous study (Münger et al., 2020), we measured the intensity of PLP, RLP, and PLS ranging from " 0 (no pain) to $10 \mathrm{~cm}$ (worst imaginable pain)". "This scale is also colored, from green (at 0 ) to red (at 10), as a visual indicator of pain. Participants are asked to rate their average pain (during an acute episode) over the last four weeks" (Münger et al., 2020). Before the participants fill each scale, an experienced researcher explained the differences between PLP, RLP, and PLS as well as any questions the participant may have about the questionnaires.

\subsection{TMS acquisition and processing}

All the TMS assessments were performed by a blinded evaluator. This trial investigated changes in cortical excitability by evaluating the motor evoked potential (MEP) and the resting motor threshold (MT). Intracortical excitability and inhibition were measured using the technique of paired-pulse, and cortical mapping. These neurophysiological variables have been evaluated in both the cerebral hemispheres: the affected hemisphere (AHM), which is contralateral to the amputated limb, and the nonaffected hemisphere (NAHM), which is ipsilateral to the amputated limb. The TMS assessments were performed with a Bistim2 stimulator using a commercially available figure 8 coil (Magstim Company LTDA, UK).

\subsubsection{Motor evoked potentials (MEPs) assessment}

Responses to stimuli applied to the motor cortex were recorded from the contralateral first dorsal interosseous (FDI) muscle. After careful consideration, the cortical representation of the hand area was chosen instead of the leg area since reliability and reproducibility is well known for these measurements in the hand area (Mortifee et al., 1994, Weiss et al., 2013). Besides that, due to the large variability in the level of lower limb amputations and the leg representation being mostly hiden in the interhemispheric fissure, choosing a target muscle in the lower limb able to represent changes in all amputees would not be possible; and would have generated a major discomfort during the recording sessions since higher intensities are necessary to elicit a response. To record MEPs, silver chloride electrodes were placed over the muscle belly (active electrode) and joint or tendon of the muscle (reference electrode). A third electrode (ground), was placed over the wrist (head of the ulnar bone). Electromyography (EMG) was processed using Powerlab 4/30 (ADinstruments, Colorado Springs, CO, USA) with a band pass filter of $20-2000 \mathrm{kHz}$. Head measures were taken to identify an approximate spot representing the primary motor cortex, using the vertex as the reference (Williams et al., 2010). The spot was used to position the TMS coil at an angle of 90 degrees with respect to the sagittal line of the head. The hotspot was determined by eliciting the highest and most stable motor evoked potential (MEP) amplitudes over the FDI (Ališauskienè et al., 2007, Temesi et al., 2014). The most accurate location was precisely marked with a pen on a swim cap that each participant wore during all the assessments. According to Rossini et al. (2015), resting motor threshold (rMT) has been determined by the smallest intensity in which 3 out of 5 motor-evoked potentials (MEPs) with minimal peak-to-peak amplitude of $100 \mu \mathrm{V}$ can be measured. After the rMT identification, 10 MEPs were recorded for each hemisphere using $120 \%$ of the rMT or higher intensities that lead to a response of at least $1 \mathrm{mV}$ (Williams et al., 2010).

\subsubsection{Paired pulse (PP) assessment}

We used the paired-pulse TMS measurements to evaluate intracortical facilitation (ICF) and short intracortical inhibition (SICI). In these types of assessments, a subthreshold conditioning stimulus ( $80 \%$ of rMT) is applied, followed by a second (suprathreshold) test stimulus (120\% of the rMT) after a variable interstimulus interval (ISI). We used the following ISIs: 2, 3, 10, and $12 \mathrm{~ms}$. Ten recordings of each inter stimulus interval protocol were randomly elicited (total of 60 measures). The subsequent offline analyses included measures of peak-to-peak amplitude of all MEPs. No neuronavigation was used.

We calculated the ICI and ICF as a ratio of the MEP (higher ICI ratio indicates less inhibition, higher ICF ratio indicates higher facilitation), according to the following formula:

$$
\begin{aligned}
\mathrm{ICI} / \mathrm{ICF} \text { ratio }= & (\text { peak }- \text { to }- \text { peak of } \mathrm{ICI} / \mathrm{ICF} * 100) / \text { peak }- \text { to } \\
& - \text { peak of MEP }
\end{aligned}
$$

\subsubsection{Cortical mapping assessment}

To assess changes in the cortical reorganization, we additionally performed the mapping of the motor cortex areas corresponding to the FDI muscle. For that, seven stimulation pulses at $120 \%$ of rMT intensity (posterior to anterior current) were delivered to 15 sites forming a $3 \times 5 \mathrm{~cm}$ grid over M1 area, separated by $1 \mathrm{~cm}$ of distance to each stimulation site, we use the hotspot (stimulation site with largest MEP) as the center of the grid (Pinto et al., 2016, Rossini et al., 2015) (see Supplementary Figure S1). The peak-to-peak amplitudes of the recorded MEPs were measured and averaged offline for each site by an assessor to establish a topographical map.

In order to evaluate the grade of excitability, a map volume in each hemisphere was calculated. Map volume is defined as the "sum of the average MEP amplitude at each location stimulated, normalized by the average MEP at the location of the largest response".

In order to calculate the spatial extent of the representation (Rossini et al., 2015), a heat map was made using MATLAB ${ }^{\circledR}$, without considering a minimal limit of amplitude (all 15 points included in this analysis). In addition, a map center of gravity $(\mathrm{CoG})$ was calculated using the grid as reference for the system of $\mathrm{X}$ and $\mathrm{Y}$ coordinates. $\mathrm{CoG}$ is defined as "weighted average of the location for the representations exceeding $50 \%$ of the maximum, in 
which weights are given by the normalized value of the representation"(Rossini et al., 2015). The following formulas were used (Pinto et al., 2016):

$$
\operatorname{CoGx}=(\Sigma x i * \mathrm{MEPi}) / \Sigma \mathrm{MEPiCoGx}=(\Sigma \mathrm{yi} * \mathrm{MEPi}) / \Sigma \mathrm{MEPi}
$$

MEPi is the mean amplitude of the MEPs in one site, while the sum of the average MEP amplitude has been calculated for each active site (site at which the mean MEP amplitude was at least $0.05 \mathrm{mV}$ ). No neuronavigation was used.

\subsection{MRI acquisition and processing}

\subsubsection{Acquisition}

All subjects were scanned at the same site; the scans were performed with a 3 Tesla Philips Achieva Scanner at the Center for Biomedical Imaging (CBI) at the Boston University Medical Center (Philips Medical Systems, Eindhoven, The Netherlands). The anatomical T1-weighted images were acquired through a turbo spin echo sequence $\left(\mathrm{TE}=3.1 \mathrm{~ms}, \mathrm{TR}=6.8 \mathrm{~ms}\right.$, flip angle $=9^{\circ}$, voxel size $0.98 \times 0.98 \times 1.20 \mathrm{~mm}$, no slice gap, acquisition matrix $256 \times 254)$.

\subsubsection{Processing}

We performed voxel-wise grey matter asymmetry analysis in statistical parametric mapping (SPM12) software (Kurth et al., 2015). The T1-scans were analyzed using the CAT12 toolbox (http://www.neuro.uni-jena.de/cat/) for SPM12 (https://www.fil. ion.ucl.ac.uk/spm/software/spm12/) in Matlab. Initially, all the images were segmented into grey matter (GM) and white matter (WM) and were also flipped using ImCalc' (by flipping). From the flipped and unflipped versions, an asymmetric DARTEL template and a right-hemispheric mask in symmetric template space in MRIcron (http://people.cas.sc.edu/rorden/mricron/index.html) were created. The right hemisphere mask enables the analysis to be performed only on one hemisphere (for flipped and unflipped images).

The asymmetry index (AI) was calculated using the formula $\mathrm{AI}=$ $\left((\mathrm{i} 1-\mathrm{i} 2) /\left((\mathrm{i} 1+\mathrm{i} 2)^{*} 0.5\right)\right) * \mathrm{i} 3$, with i1 being the warped non-flipped images, i2 being the warped flipped images, and i3 being the right-hemispheric mask. Only after the calculation, the AI grey matter images were smoothed using an $8 \mathrm{~mm}$ smoothing kernel as suggested by the Luders 2015 guidelines (Kurth et al., 2015). In order to simplify the comparison between affected (hemisphere contralateral to the amputated side) and nonaffected hemisphere, subjects with the left hemisphere affected (right lower limb amputation) had their AI images multiplied by -1 using ImCalc'. Therefore, all AI images are presenting the differences between affected ("right") versus the nonaffected hemisphere ("left"). Consequently, in our analysis, negative AIs values indicated larger nonaffectedhemispheric grey matter volume, while positive AIs indicated larger affected-hemispheric grey matter volume. For statistical comparisons, we used cluster-level correction (cluster extend expected) and an alpha level of 0.05 . In order to avoid spurious findings driven by noise, we applied a cluster extent threshold of 40 voxels (Kurth et al., 2015). Anatomical locations of significant clusters were determined using their MNI coordinates in MRIcron and xjview using the AAL template.

\subsubsection{Neurosynth activation mask and decoding}

Neurosynth is a public database that lists the results from $>14000$ functional MRI published investigations (https://neurosynth.org/). Neurosynth can tag individual studies with all words that occur at least once in its abstract, excluding "stopwords" and words that occur in more than $60 \%$ of all abstracts, therefore studies can be retrieve using a specific term. We used Neurosynth's to create an activation mask based on the terms "hand" and "foot", by simply typed the term "hand" a statistical map of an automated meta-analysis is generated; For the term "hand" there were 879 studies, and for the term "foot" there were 83 studies. Using Neurosynth's we were able to create a generalizable mask, dependent on the activation of this regions in several studies, instead of using only an atlas generate mask. We used a T-threshold of 5.5 and exported these maps into NIFTI format, where they were used in further analysis as ROI masks (Yarkoni et al., 2011).

\subsection{Statistical analysis}

We used complete case analysis to manage missing data. When the data was not symmetrically distributed, we used median and interquartile range to reduce the impact of outliers. The ShapiroWilk's test, skewness, and kurtosis were used to assess normality. We reported baseline characteristics using central tendency and dispersion measurements based on the variable type (e.g., mean and SD for continuous variables, frequency tabulations for categorical variables). To test groups or clusters differences, we used Mann-Whitney-U-Tests and for correlations Spearman Rank and Pearson's Correlation Tests. The statistical analyses were performed with Stata version 15.1.

\section{Results}

\subsection{Single and Paired-pulsed TMS}

All the TMS variables were approximately normally distributed. Regarding the MEP, SICI, and ICF among affected and nonaffected hemisphere, there was no statistically significant difference (see Table 2).

\subsection{Cortical mapping}

We found an anterior shift of the hand area "hotspot" in the affected hemisphere ( $23 \mathrm{~mm}, 95 \% \mathrm{CI}: 0.07$ to $0.38, \mathrm{p}=0.006)$, see Fig. $1 \mathrm{~A}$. The lateral axis and the volume (affected $=4.56 \pm 1.80$; non-affected $=4.33 \pm 1.60$ ) did not differ statistically, among affected and nonaffected hemispheres, although the volume was bigger in the affected hemisphere (see Table 2). After controlling by use of medications (opioids, gabapentin, pregabalin, nonopioids analgesics, and antidepressants), our findings on does not change.

In the 3D representation of the grid (Fig. 1B), we can see a predominant peak of excitability (COG) together with a disorganized and widespread secondary peaks in the representation of the hand cortical in the motor cortex of the affected hemisphere.

The Supplementary Figure S2 shows a matrix representation with the means and SDs of each stimulation point per hemisphere.

Table 2

Transcranial magnetic stimulation (TMS) findings $(n=62)$.

\begin{tabular}{lll}
\hline TMS variables & $\begin{array}{l}\text { Affected hemisphere } \\
(\text { Mean } \pm \text { SD) }\end{array}$ & $\begin{array}{l}\text { Nonaffected hemisphere } \\
\text { (Mean } \pm \text { SD) }\end{array}$ \\
\hline MEP $(\mathrm{mV})$ & $1.44 \pm 0.84$ & $1.58 \pm 0.84$ \\
ICI $(\%)$ & $0.56 \pm 0.36$ & $0.58 \pm 0.36$ \\
ICF $(\%)$ & $1.42 \pm 0.56$ & $1.52 \pm 0.71$ \\
Volume $\left(\mathrm{mV}^{2} \mathrm{x} \mathrm{cm}\right)$ & $4.56 \pm 1.80$ & $4.33 \pm 1.60$ \\
COG $\times$ coordinate $(\mathrm{mm})$ & $1.02 \pm 0.34$ & $1.10 \pm 0.35$ \\
COG y coordinate $(\mathrm{mm})$ & $2.20 \pm 0.46$ & $1.97 \pm 0.42$ \\
\hline
\end{tabular}

Abbreviations: motor evoked potential (MEP), intracortical inhibition (ICI), intracortical facilitation (ICF), center of gravity (COG). 

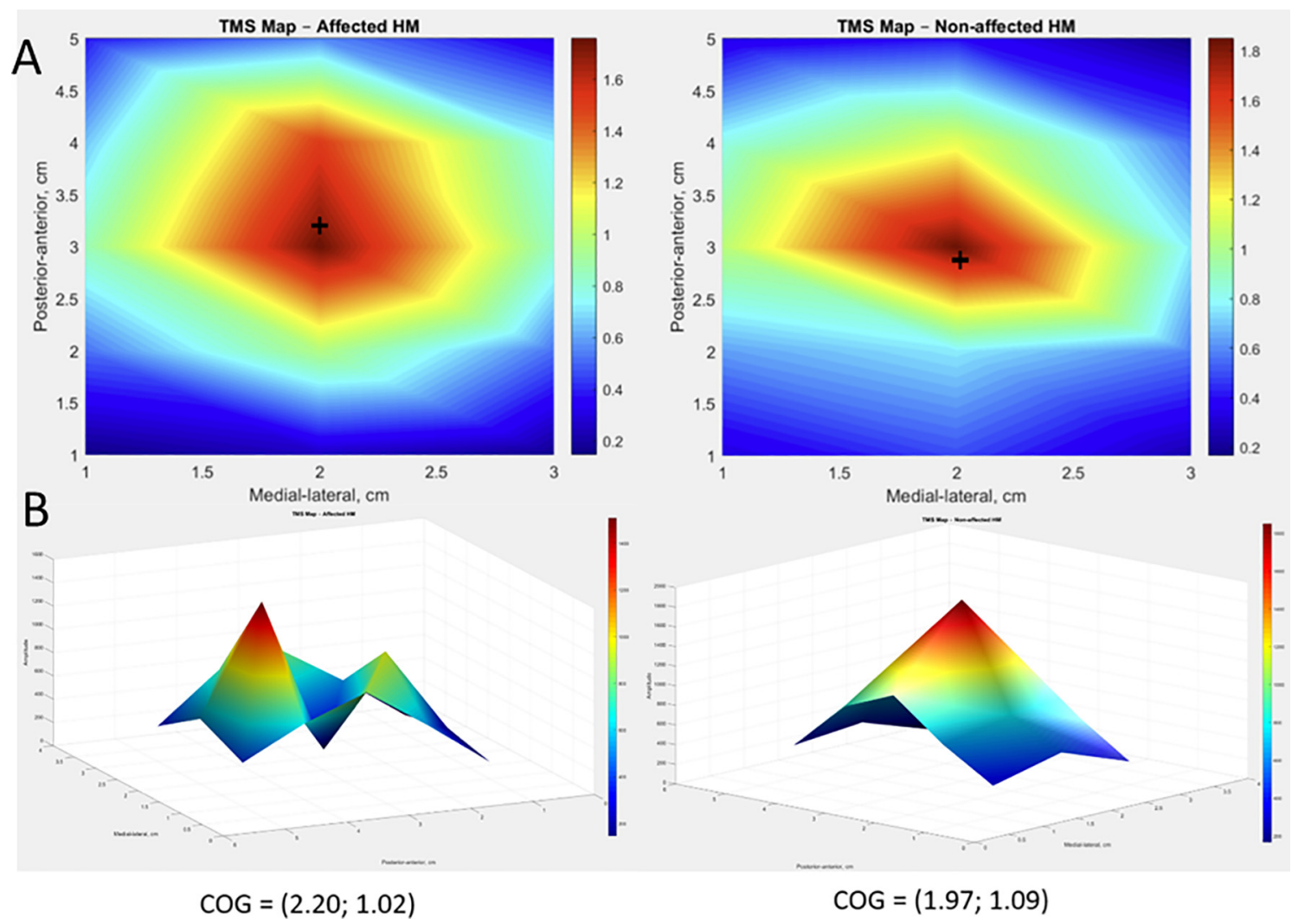

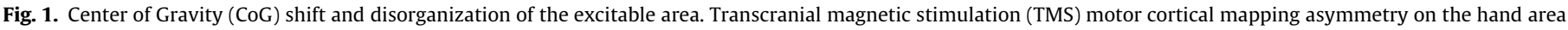

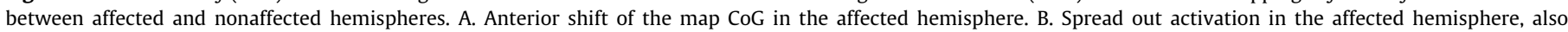

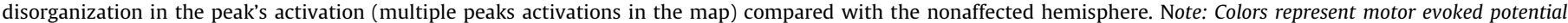
(MEP) amplitudes (red = high amplitude).

\subsection{Structural grey matter asymmetries between affected and nonaffected hemispheres}

Twenty-one subjects were included in the VBM analysis, a subset of the Boston site participants. For the analysis, we first setup two statistical models using a one-sample t-test (cluster extent threshold $=40, \mathrm{p}<0.001$, FWE-uncorrected) to check for clusters with asymmetry index significantly different from zero; one contrast was performed for the positive AIs and the other for negative AIs using SPM12. Overall, there were no clusters surviving cluster extend analysis for negative or positive AIs in sensory-motor areas, indicating no statistical differences when comparing affected and nonaffected hemisphere grey matter in PLP participants.

\subsection{Asymmetry differences in activation mask}

We use a Neurosynth to create an activation mask for the motor cortex areas using the terms "hand" and "leg". This analysis was performed to identify possible asymmetry differences in these two regions that, even though did not survive the cluster extend analysis (due to the small sample size and multiple comparison corrections), could potentially help to explain the motor cortex activation differences observed in the TMS assessments. In this exploratory analysis, for both regions, the median $\mathrm{AI}$ is negative, which means that the grey matter volume is decreased in the affected hemisphere compared with the nonaffected (Fig. 2A). Also, we found that subjects with two or more years since amputation present a higher AI (although no statistical significant), and the asymmetry index is closer to zero (i.e., less negative, diff $=0.177$ for the hand area and diff $=0.0437$ for the leg area) meaning the affected hemisphere has similar grey matter compared with nonaffected in these subjects (Fig. 2B).

\subsection{Correlational analysis}

The univariate analysis found that PLP intensity is negatively correlated with ICF ratio in the affected hemisphere $(r=-0.25$, $\mathrm{p}=0.04)$ - less PLP intensity is correlated with higher ICF. Also, age $(r=-0.27, p=0.04)$ and time since amputation $(r=-0.42$, $\mathrm{p}=0.007$ ) are negatively correlated with $\mathrm{SICI}$ in the affected hemisphere (older patients and longer time since amputation are correlated with higher inhibition). Other findings are the SICI ratios of both hemispheres (affected versus nonaffected) are positively correlated $(\mathrm{r}=0.5, \mathrm{p}=0.001)$ - the inhibition in the affected hemisphere is correlated with the inhibition in the nonaffected. Also, we found a positive intra-hemisphere correlation among SICI and ICF in the affected hemisphere $(r=-0.35, p=0.006)$ and nonaffected hemisphere $(r=-0.32, p=0.01)$ - meaning that less inhibition is correlated with higher facilitation in the same hemisphere.

The PLP intensity was not associate with the use of pain medication (opioids, gabapentin, pregabalin, non-opioids analgesics, and antidepressants), neither the time since amputation, or the protheses use (protheses users: plp mean $=5.79, \mathrm{SD}=1.64$; protheses non-users: plp mean $=6.36, \mathrm{SD}=1.81$ ), although there is a trend of less pain in protheses users.

Regarding the VBM analysis, there was a significantly negative correlation $(\mathrm{r}=-0.50, \mathrm{p}=0.032)$ between volume in the affected hand representation area (hand asymmetry index) with the MEP amplitude in the same area; therefore, less volume correlated with increased MEP amplitude. Finally, the level of amputation was not associate with the degree of COG anterior shift (difference $=0.07$, 

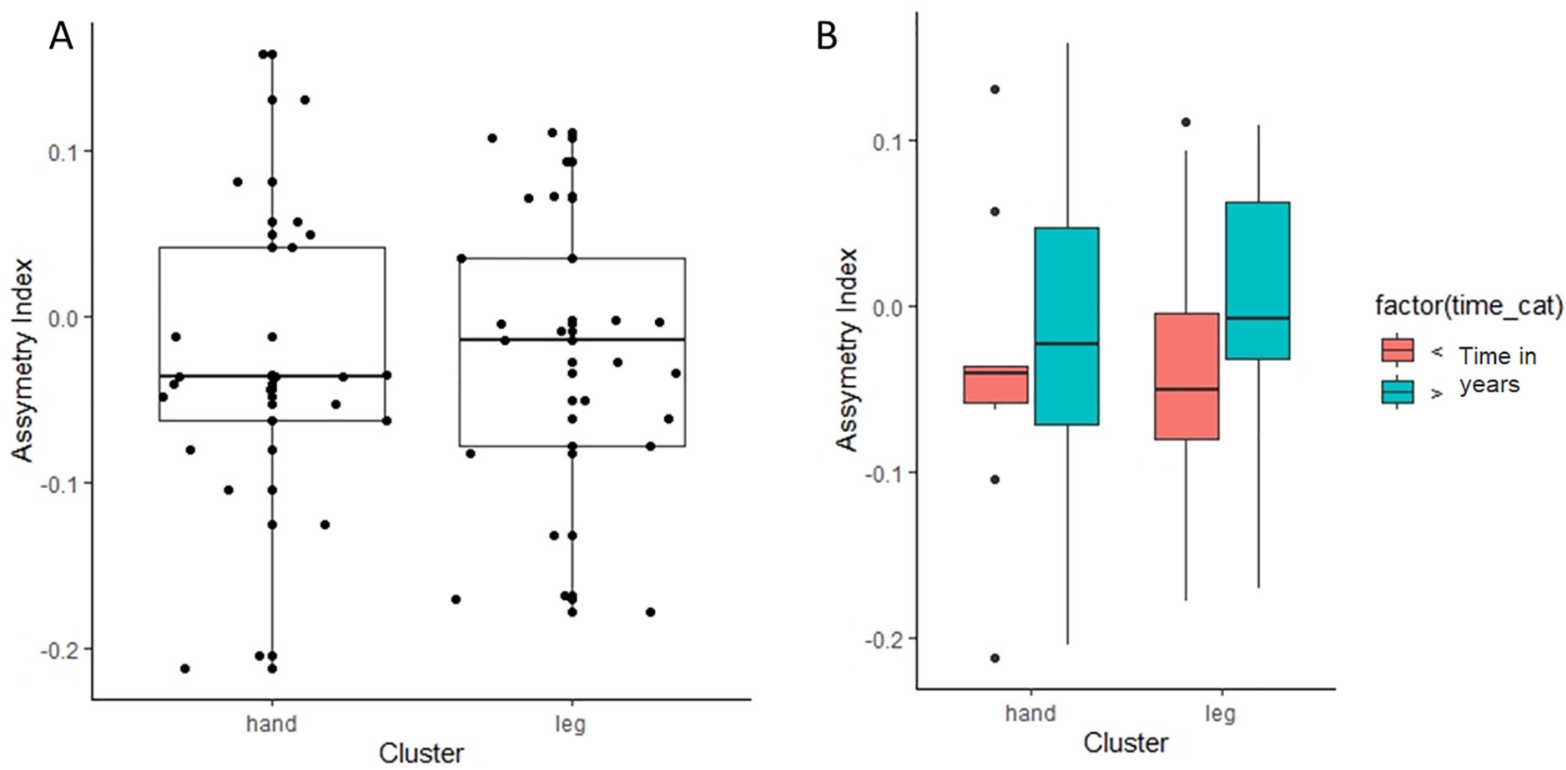

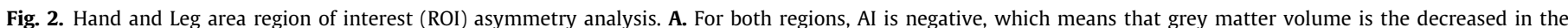

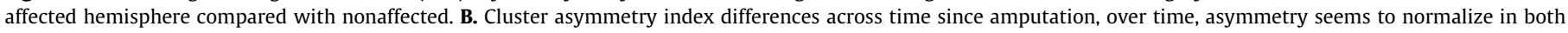
regions. In the boxplots, the median and the interquartile range are represented, the whiskers show the lowest and highest observations.

$\mathrm{p}=0.64$ ) or grey matter asymmetry in the hand (difference $=0.045$, $\mathrm{p}=0.32$ ) or leg areas (difference $=-0.013, \mathrm{p}=0.75$ ).

\section{Discussion}

In this study, we evaluated the neurophysiological and structural motor cortex asymmetry in lower limb amputees with phantom limb pain, indexed by two different techniques (TMS and MRI); and how these correlates with clinical parameters of phantom limb pain. We showed a statistically significant anterior shift of the hand area center of gravity $(\mathrm{CoG})$ in the affected hemisphere. Besides that, the hand cortical representation in the affected motor cortex was disorganized and more widespread than the nonaffected one. For the MRI asymmetry analysis, we found no statically significant cluster in the sensory-motor areas. However, in the hand and leg areas, we showed that there is a loss of volume in the affected hemisphere when compared with the non-affected, that seems to be negatively correlated with time since amputation and increased MEP amplitudes. Only the ICF ratio in the affected hemisphere is correlated with PLP intensity.

\subsection{Correlation with pain intensity}

In our study, we showed evidence of asymmetrical neurophysiological and structural motor cortex reorganization. However, our results suggest a dissociation between CoG shift and intensity of pain since we showed a difference in the CoG between the hemisphere but no correlation with pain intensity in lower limb amputees. Moreover, we also showed a loss of grey matter volume in the motor area representation of the "hand" that was negative correlated with MEP intensity - although we cannot say it is the same area where the TMS assessment was performed due to the lack of neuronavigation - but showed no relation with pain levels. Interestingly, a recent systematic review (Gunduz et al., 2019), also found similar results - changes in cortical mapping not correlated with pain intensity - while analyzing cortical mapping organization using TMS.

Although most of our findings were not correlated with PLP intensity, it may be related to the presence of pain. Previous studies showed different changes in gray matter for patients with High vs. Low intensity of PLP (Preißler et al., 2012); however, our sample did not include patients with low-intensity PLP or without pain. We hypothesized that the amount of reorganization could be associated with the presence of PLP instead of its severity. Indeed, studies showed reduced gray matter in the motor cortex area representing the amputated limb, but there were no correlations with pain intensity (Preißler et al., 2012). Correspondingly, only patients with PLP (upper limb amputees) presented an increase of the mapped area for the muscles above the stump and medial displacement of the CoG of the adjacent mouth area in the affected hemisphere (Karl et al., 2001). One potential contribution from our study is to provide additional support reinforcing the notion that PLP intensity may be more related to other neural circuits such as those related to emotional processing (Münger et al., 2020). In other words, reorganization may provide the necessary changes to trigger pain circuits to be functionally more active. Then circuits related to emotional-affective processing may be critical ones to determine pain intensity.

PLP has been attributed to a maladaptive reorganization of the primary somatosensory (S1) and motor (M1) cortex. Specifically, studies have suggested that PLP is related to changes in the activity of intracortical inhibitory and excitatory interneuronal circuits, as a reduction in SICI and an enhancement of ICF at the hemisphere contralateral to the amputation. The precise mechanisms underlying the development of pain in patients with limb amputation are not well elucidated, and current evidence shows inconsistencies to support the relationship between PLP and imbalanced cortical activity (Gunduz et al., 2019).

We found that ICF is negatively correlated with PLP, it is aligned with previous literature showing that the activation of the motor cortex (higher ICF) generated by anodal tDCS contributed with its analgesic effect (Biabani et al., 2017). In the same way, rTMS studies have been showing that the lack of inhibition in the motor cortex is an important mechanism of PLP. Additional evidence has shown increased cortical excitability (larger motor-evoked potentials and decreased intracortical inhibition) in areas corresponding to the stump muscles. This increased excitability after amputation is associated with the down-regulation of gamma-aminobutyric acid (GABA)-related inhibitory circuits (Nardone et al., 2019). 
Schwenkereis et al. (2000) demonstrated after upper limb amputation that ICF differed significantly from the other hemisphere and from the control group (non-amputees). Regarding SICI, no significant difference was found in upper arm amputees neither between hemispheres nor between one of these sides and the control group. The authors suggested an enhancement of corticocortical excitability observed in the contralateral hemisphere to the amputation, which differed according to the level of amputation. In another study, an NMDA antagonist (memantine) treatment for 3 weeks reduced ICF and enhanced SICI; however, these changes were not correlated to the reduction of phantom pain. Therefore, the authors conclude that NMDA-mediated mechanisms influence changes of cortical activity occurring after limb amputation; however these cortical excitability changes and phantom pain are independent of each other (Maier et al., 2003).

\subsection{Brain changes after amputation}

In the motor areas, we showed that there is a loss of volume in the $\mathrm{AH}$ (hand and leg areas), that seems to be related with time since amputation. In addition, the SICI in the AH is negatively correlated with the time since amputation.

It is well established that, in the post-amputation, there is an acute lack or disruption of afferent inputs that were originally presented from the missing limb. This disruption exerts central reorganization changes that remain not well established, as multiple studies showed contradictory findings. Among the most relevant data, a loss or reduction of volume of the corresponding area in the brain to the amputated limb, in particular, a decrease in grey matter in either motor or sensory cortex, is commonly reported (Draganski et al., 2006, Jiang et al., 2015, Preißler et al., 2012). From a theoretical standpoint, it is understandable that the lack of afferent inputs can lead to a decrease in the volume of certain areas the same way a muscle size decreases due to a lack of usage. In this regard, several studies have shown similar findings to the ones seen in our analysis. Di vita et al. (2018)(Di Vita et al., 2018) revealed alterations on the cerebellar grey matter in amputees that did not use prosthesis - lack of any type of afferent input -, the most frequent finding was a significant reduction of the grey matter in sensory-motor cerebral areas corresponding to the missing limb. In addition, supporting evidence revealed that lower limb amputees using prosthesis had no reduction in the grey matter volume when compared with healthy controls in contrast to a significant decrease in the grey matter of amputees that did not use a prosthesis. The prior findings once more support the theory in which a lack of afferent inputs causes some degree of atrophy/central reorganization in the grey matter of the affected areas. There is a gap in evidence regarding the alteration of white matter, although we could expect that following the same premise, axonal tracks that are underutilized could be affected hence leading to a decrease of white matter in amputees. Further prospective studies are needed to evaluate asymmetrical white matter alterations in PLP patients.

Another relevant aspect associated to the degree of central organization is the affected area, as it is well-known centers of control in charge of more specialized functions have greater representations in the brain - ex: larger hand or face representation in contrast to truncal or lower limb representation. This was confirmed by Jiang et al. (2015) while evaluating lower limb amputees showing no significant changes in the volume of the grey matter, in contrast to other well-established findings by Preißler et al. (2012) of a marked decrease of grey matter volume corresponding to the left-hand area.

Besides the motor areas, regional decreases in grey matter volume in other cortical and subcortical regions are widely documented in chronic pain (Rodriguez-Raecke et al., 2009), and commonly argued to be a consequence of pain presence (not related with intensity).

Even though the exact mechanism leading to this grey matter decrease and structural changes remains yet to be elucidated; in the case of amputees and our results, the lack of afferent inputs to one region - leg area of the motor cortex; may lead to the unmasking of existing synapses carrying similar information to neighboring areas - i.e. hand area - enable by the increased motor cortex disinhibition and can partially explain the changes in cortical mapping and grey matter volume observed in the hand area (Graziano and Jones, 2009). Although, it is important to point out that the VBM analysis did not take in consideration the possible shift in the hand area representation on the affected hemisphere; however the mask used for the hand area identification was created based on a meta-analysis including 879 studies making it very generalizable.

In the same way, increases of motor evoked potential (MEP) can reflect the dysfunction of motor cortex interconnections that are known to be mainly mediated by inhibitory responses. Moreover, an increase in motor cortex activation may have a role in the adaption to the dysfunction damage within the motor cortex, therefore, explaining the correlation between more dysfunction (reduced grey matter) and increase MEP response showed in the hand areas in our study. Alternatively, this stronger volumetric decline could be related to the overactivation and motor cortex specialization (efficiency network theory), similarly to high performance athletes with small but highly effective motor representation.

Besides that, other mechanisms such as simple cell atrophy, synaptic reorganization or decrease in cell body size would have to be explored to better understand the decreases in grey matter volume in the affected hemisphere and if the relationship between volume and activity can be a results of inter-subject variability due to hand use - specializing/mastering skills of a determined structures/limbs leads to smaller brain representations of that area -; alternatively, a feedback mechanism following deafferentation, or a consequence of motor dysfunction related to increase use of upper limb, while decreased usage of the lower limb can lead to the opposite. The interpretation of this result is particularly challenging due to the lack of dominant handedness information; therefore, we cannot exclude the possibility of the differences in volume and motor evoked potential to be a result of differences in hand use/pattern.

Our findings are of extreme interest as we were able to show a decrease in volume in the affected hemisphere that balances/ reestablishs over time despite of pain levels in these subjects. This may indicate a more permanent functional condition (i.e., phantom limb pain) despite of a normalization of the anatomical signature (i.e., grey volume).

\subsection{Limitations and strengths}

We did not have a control population of amputee's subjects that do not have PLP, so it is not possible to make certain comparisons to know at which level the presence of pain could be truly correlated with the level of cortical disorganization. Another limitation is that we used the hand area instead of the leg area in the TMS assessment, this was done mainly because the leg area is difficult to achieve while doing TMS and as our main hypothesis was to look if there was the cortical reorganization, thus this is possible assessing the hand area (Bungert et al., 2016). Also, we do not have handedness data from the participants, however, in our sample the proportion of left amputees is higher than right amputees (56\% vs. $43 \%$ ) and considering that more than $93 \%$ of the population are right-handed, we do not expect important effect of this variable in our results (Coren and Porac, 1977). As well, this was a crosssection analysis, making it impossible to determine the real tempo- 
ral relation between cortical disorganization and levels of pain. Since we are using MRI results from a subset of participants, it is essential to mention that TMS and MRI findings cannot be compared to each other directly. Besides, due to the lack of spatial resolution of the TMS mapping and the absence of neuronavigation aid, we cannot precise an anatomical location of the reported shift, however, we can state that a cortical reorganization phenomenon is present in the affected hemisphere, Finally, since our correlation analysis are exploratory, we did not correct by multiple comparisons, thus our results needing confirmation in larger samples with proper correction. One important strength to underscore is the relatively large sample size for a homogeneous population: traumatic and single lower limb amputation.

\section{Declaration of interest}

The authors report no conflict of interest.

\section{Acknowledgments}

This work is supported by a NIH grant (R01-HD082302-01A1).

\section{Appendix A. Supplementary material}

Supplementary data to this article can be found online at https://doi.org/10.1016/j.clinph.2020.06.024.

\section{References}

Ališauskienė M, Magistris MR, Vaičienė N, Truffert A. Electrophysiological evaluation of motor pathways to proximal lower limb muscles: a combined method and reference values. Clin Neurophysiol: Off J Int Feder Clin Neurophysiol 2007;118(3):513-24.

Biabani M, Aminitehrani M, Zoghi M, Farrell M, Egan G, Jaberzadeh S. The effects of transcranial direct current stimulation on short-interval intracortical inhibition and intracortical facilitation: a systematic review and meta-analysis. Rev Neurosci 2017;29(1):99-114.

Birbaumer N, Lutzenberger W, Montoya P, Larbig W, Unertl K, Töpfner S, et al. Effects of regional anesthesia on phantom limb pain are mirrored in changes in cortical reorganization. J Neurosci 1997;17(14):5503-8.

Bungert A, Antunes A, Espenhahn S, Thielscher A. Where does TMS stimulate the motor cortex? Combining electrophysiological measurements and realistic field estimates to reveal the affected cortex position. Cereb Cortex 2016;27 (11):5083-94.

Cohen LG, Bandinelli S, Findley TW, Hallett M. Motor reorganization after upper limb amputation in man: a study with focal magnetic stimulation. Brain 1991;114(1):615-27.

Coren S, Porac C. Fifty centuries of right-handedness: the historical record. Science 1977;198(4317):631-2.

Di Vita A, Boccia M, Palermo L, Nemmi F, Traballesi M, Brunelli S, et al. Cerebellar grey matter modifications in lower limb amputees not using prosthesis. Sci Rep 2018;8(1):370.

Draganski B, Moser T, Lummel N, Gänssbauer S, Bogdahn U, Haas F, et al. Decrease of thalamic gray matter following limb amputation. Neuroimage 2006;31 (3):951-7.

Flor H. Phantom-limb pain: characteristics, causes, and treatment. The Lancet Neurology 2002;1(3):182-9.

Flor H, Elbert T, Knecht S, Wienbruch C, Pantev C, Birbaumers N, et al. Phantom-limb pain as a perceptual correlate of cortical reorganization following arm amputation. Nature 1995;375(6531):482.

Flor H, Nikolajsen L, Jensen TS. Phantom limb pain: a case of maladaptive CNS plasticity?. Nat Rev Neurosci 2006;7(11):873.

Gagné M, Hétu S, Reilly KT, Mercier C. The map is not the territory: motor system reorganization in upper limb amputees. Hum Brain Mapp 2011;32(4):509-19.

Graziano A, Jones EG. Early withdrawal of axons from higher centers in response to peripheral somatosensory denervation. J Neurosci 2009;29(12):3738-48.

Grüsser SM, Winter C, Mühlnickel W, Denke C, Karl A, Villringer K, et al. The relationship of perceptual phenomena and cortical reorganization in upper extremity amputees. Neuroscience 2001;102(2):263-72.

Gunduz ME, Pinto CB, Velez FGS, Duarte D, Pacheco-Barrios K, Lopes F, et al. Motor cortex reorganization in limb amputation: a systematic review of TMS motor mapping studies. Front Neurosci 2019;14(314):1-11.

Jiang G, Yin X, Li C, Li L, Zhao L, Evans AC, et al. The plasticity of brain gray matter and white matter following lower limb amputation. Neural Plast 2015;2015:1-10.
Karl A, Birbaumer N, Lutzenberger W, Cohen LG, Flor H. Reorganization of motor and somatosensory cortex in upper extremity amputees with phantom limb pain. J Neurosci 2001;21(10):3609-18.

Kikkert S, Johansen-Berg H, Tracey I, Makin TR. Reaffirming the link between chronic phantom limb pain and maintained missing hand representation. Cortex 2018;106:174-84.

Kooijman CM, Dijkstra PU, Geertzen JHB, van der Schans CP, Elzinga A. Phantom pain and phantom sensations in upper limb amputees: an epidemiological study. Pain 2002;87(1):33-41.

Kurth F, Gaser C, Luders E. A 12-step user guide for analyzing voxel-wise gray matter asymmetries in statistical parametric mapping (SPM). Nat Protoc 2015:10(2):293.

MacIver K, Lloyd DM, Kelly S, Roberts N, Nurmikko T. Phantom limb pain, cortical reorganization and the therapeutic effect of mental imagery. Brain 2008;131 (8):2181-91.

Maier C, Dertwinkel R, Mansourian N, Hosbach I, Schwenkreis P, Senne I, et al. Efficacy of the NMDA-receptor antagonist memantine in patients with chronic phantom limb pain-results of a randomized double-blinded, placebo-controlled trial. Pain 2003:103(3):277-83.

Makin TR, Scholz J, Filippini N, Slater DH, Tracey I, Johansen-Berg H. Phantom pain is associated with preserved structure and function in the former hand area. Nat Commun 2013;4:1570.

Montoya P, Ritter K, Huse E, Larbig W, Braun C, Töpfner S, et al. The cortical somatotopic map and phantom phenomena in subjects with congenital limb atrophy and traumatic amputees with phantom limb pain. Eur J Neurosc 1998;10(3):1095-102.

Mortifee P, Stewart H, Schulzer M, Eisen A. Reliability of transcranial magnetic stimulation for mapping the human motor cortex. Electroencephalography Clin Neurophysiol/Evoked Potent Section 1994;93(2):131-7.

Münger M, Pinto CB, Pacheco-Barrios K, Duarte D, Gunduz ME, Simis M, et al. Protective and risk factors for phantom limb pain and residual limb pain severity. Pain Practice 2020;20(5):460-70.

Nardone R, Versace V, Sebastianelli L, Brigo F, Christova M, Scarano GI, et al. Transcranial magnetic stimulation in subjects with phantom pain and nonpainful phantom sensations: a systematic review. Brain Res Bull 2019.

Nikolajsen L, Jensen TS. Phantom limb pain. Br J Anaesth 2001;87(1):107-16.

Pinto CB, Velez FGS, Bolognini N, Crandell D, Merabet LB, Fregni F. Optimizing rehabilitation for phantom limb pain using mirror therapy and transcranial direct current stimulation: a randomized, double-blind clinical trial study protocol. JMIR Res Protcols 2016:5(3) e138.

Preißler S, Feiler J, Dietrich C, Hofmann GO, Miltner WHR, Weiss T. Gray matter changes following limb amputation with high and low intensities of phantom limb pain. Cereb Cortex 2012;23(5):1038-48.

Preißler S, Thielemann D, Dietrich C, Hofmann GO, Miltner WHR, Weiss T. Preliminary evidence for training-induced changes of morphology and phantom limb pain. Front Hum Neurosci 2017;11:319.

Raffin E, Richard N, Giraux P, Reilly KT. Primary motor cortex changes after amputation correlate with phantom limb pain and the ability to move the phantom limb. Neuroimage 2016;130:134-44.

Ramachandran VS, Rogers-Ramachandran D, Stewart M, Pons TP. Perceptual correlates of massive cortical reorganization. Science 1992;258:1159-

Richardson C. Phantom limb pain; prevalence, mechanisms and associated factors. In: Murray C (editor). Amputation, Prosthesis Use, and Phantom Limb Pain Springer. 2009. p. 137-56.

Rodriguez-Raecke R, Niemeier A, Ihle K, Ruether W, May A. Brain gray matter decrease in chronic pain is the consequence and not the cause of pain. J Neurosci 2009;29(44):13746-50.

Rossini PM, Burke D, Chen R, Cohen LG, Daskalakis Z, Di Iorio R, et al. Non-invasive electrical and magnetic stimulation of the brain, spinal cord, roots and peripheral nerves: basic principles and procedures for routine clinical and research application. An updated report from an IFCN Committee. Clin Neurophysiol 2015;126(6):1071-107.

Schwenkreis P, Witscher K, Janssen F, Dertwinkel R, Zenz M, Malin J-P, et al Changes of cortical excitability in patients with upper limb amputation. Neurosci Lett 2000;293(2):143-6.

Sinha R, van den Heuvel WJA, Arokiasamy P. Factors affecting quality of life in lower limb amputees. Prosthet Orthot Int 2011;35(1):90-6.

Temesi J, Gruet M, Rupp T, Verges S, Millet GY. Resting and active motor thresholds versus stimulus-response curves to determine transcranial magnetic stimulation intensity in quadriceps femoris. J NeuroEng Rehabil 2014;11(1):40.

van der Schans CP, Geertzen JHB, Schoppen T, Dijkstra PU. Phantom pain and health-related quality of life in lower limb amputees. J Pain Symptom Manage 2002;24(4):429-36.

Weiss C, Nettekoven C, Rehme AK, Neuschmelting V, Eisenbeis A, Goldbrunner R, et al. Mapping the hand, foot and face representations in the primary motor cortex-retest reliability of neuronavigated TMS versus functional MRI Neuroimage 2013;66:531-42.

Williams JA, Pascual-Leone A, Fregni F. Interhemispheric modulation induced by cortical stimulation and motor training. Phys Ther 2010;90(3):398-410.

Yarkoni T, Poldrack RA, Nichols TE, Van Essen DC, Wager TD. Large-scale automated synthesis of human functional neuroimaging data. Nat Methods 2011;8(8):665. 